\title{
Chemical Characteristics of Bottom Sediments in the Colorado River Upstream from the Price-Stubb Diversion Dam near Palisade, Colorado, October-November 1998
}

\section{INTRODUCTION}

The Colorado River from Palisade upstream to Rifle, Colorado, is a historical habitat for native fish. Some of these fish, the Colorado River pikeminnow and the razorbacked sucker, are currently on the threatened and endangered list. Irrigation diversion dams built in the early 1900's have effectively cut off fish migration in the Colorado River upstream from Palisade. The Price-Stubb diversion dam is located just upstream from Palisade (fig. 1) and currently is not in use. Removal of the Price-Stubb diversion dam has been proposed by the Bureau of Reclamation (BOR) to aid in the recovery efforts of the Colorado pikeminnow and razorbacked sucker by allowing access to historical habitat for spawning.

Removal of the dam could release fine sediments $(<0.0625 \mathrm{~mm})$ that have been stored upstream from the dam into the Colorado River. There is no information available about the chemical characteristics of these bottommaterial sediments. Because the Colorado River downstream from Palisade through the Grand Valley has been designated as critical habitat for recovery of the Colorado pikeminnow, the release of the sediments and possibly toxic elements in interstitial water associated with removal of the dam is a concern of the BOR. Municipal drinkingwater providers downstream are also concerned that release of the sediments and possibly toxic elements could create a public health hazard.

Information derived from the characterization of these bottom-material sediments and associated interstitial waters is needed for planning to avoid any potential damage to threatened and endangered fish or municipal drinking-water systems. Consequently, bottom-sediment samples were collected by the U.S. Geological Survey as part of a cooperative program with the BOR. The sampling sites and data-collection activities and the chemical characteristics are described in the following sections of the report.

\section{SAMPLING SITES AND DATA COLLECTION}

Field reconnaissance determined that the finer bottom materials were located along both banks of the Colorado River from 100 to 300 feet upstream from the diversion dam and ranged in depth from 2 to 20 inches. The locations of the deeper deposits of fine material were scattered and very random. Core samples of bottom-

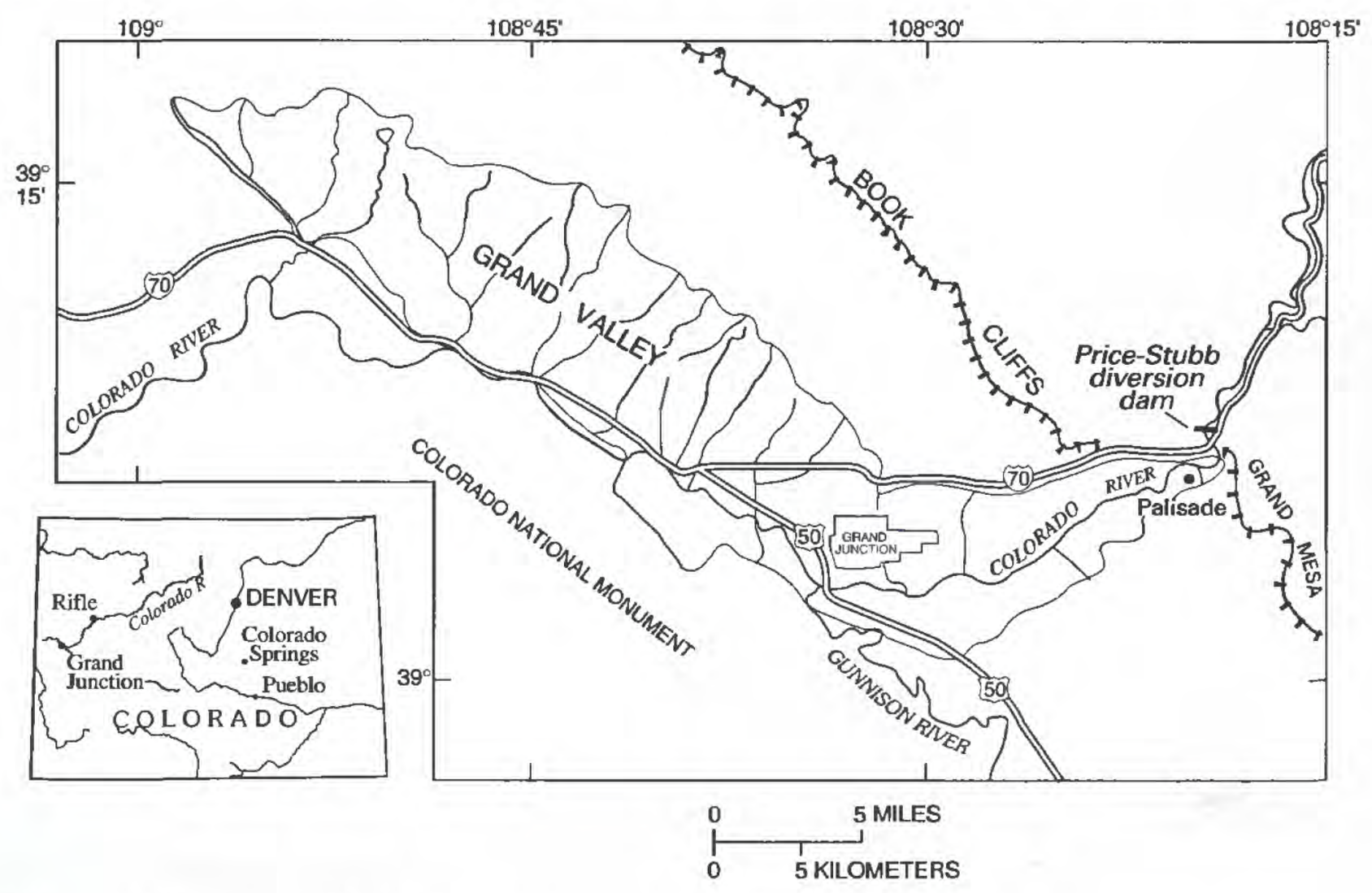

Figure 1. Location of the Grand Valley and the Price-Stubb diversion dam. 
material sediments were collected at four sites-two along the left bank and two along the right bank-during October and November 1998 (fig. 2). The core samples were taken from depths between 2.4 and 9.8 inches.

It is possible that other layers of fines exist below the streambed, but due to the composition of most of the streambed material (coarse cobbles and boulders) it was not possible to sample at greater depths. Chemical characteristics of fine material that may be present between the existing streambed and pre-dam streambed are unknown.

Attempts to sample interstitial water from the bottom sediments were unsuccessful because of the fine-grained material present.

Site PSDG1 is about 50 feet upstream from the end of the diversion wall on the right bank and 10 feet streamward. Site PSDG2 is on the right bank about 200 feet upstream from the end of the diversion wall and about 8 feet streamward. Site PSDG3 is on the left bank about 200 feet upstream from the diversion dam and about 10 feet streamward. Site PSDG4 is along the left bank about 150 feet upstream from PSDG3 and about 6 feet streamward. An additional sample, PSDG4a, was collected near site PSDG4 and analyzed for quality-control purposes.

\section{CHEMICAL CHARACTERISTICS OF BOTTOM SEDIMENTS}

Each core sample was analyzed at incremental depths for 28 constituents at the USGS sediment partitioning labor- tory. Results of the analyses are listed in table 1 . As a quality-control procedure, duplicate analyses were performed on parts of the samples at sites PSDG1 and PSDG3. Results of the chemical analyses were compared to data for reference samples that have been collected across the United States. These reference samples were selected for comparison because they were about the same sediment size and chemical composition as the core samples.

The reference sample from the Cody Shale (SCO-1) is from a site in Wyoming. It is typical of Upper Cretaceous silty marine shales, intermediate between fine-grained offshore marine shales and coarser nearshore marine siltstone and sandstone. The reference sample for shale (SDO-1) is from the Huron Member of the Ohio Shale in Kentucky. It can be used to establish analytical accuracy in the analysis of organic- and sulphur-rich sedimentary rocks. The reference sample for marine sediment (MAG-1) is a fine-grained, gray-brown, clayey mud from the Gulf of Maine. The reference sample NIST 2709 is agricultural soil from the San Joaquin Valley in California.

The reference samples can be used to establish background values of different constituents. The comparison of concentrations between the reference samples and the core samples indicated that the bottom sediments upstream from the Price-Stubb diversion dam are at the same level or less than the reported values of the reference samples. No unusually high concentrations were determined.

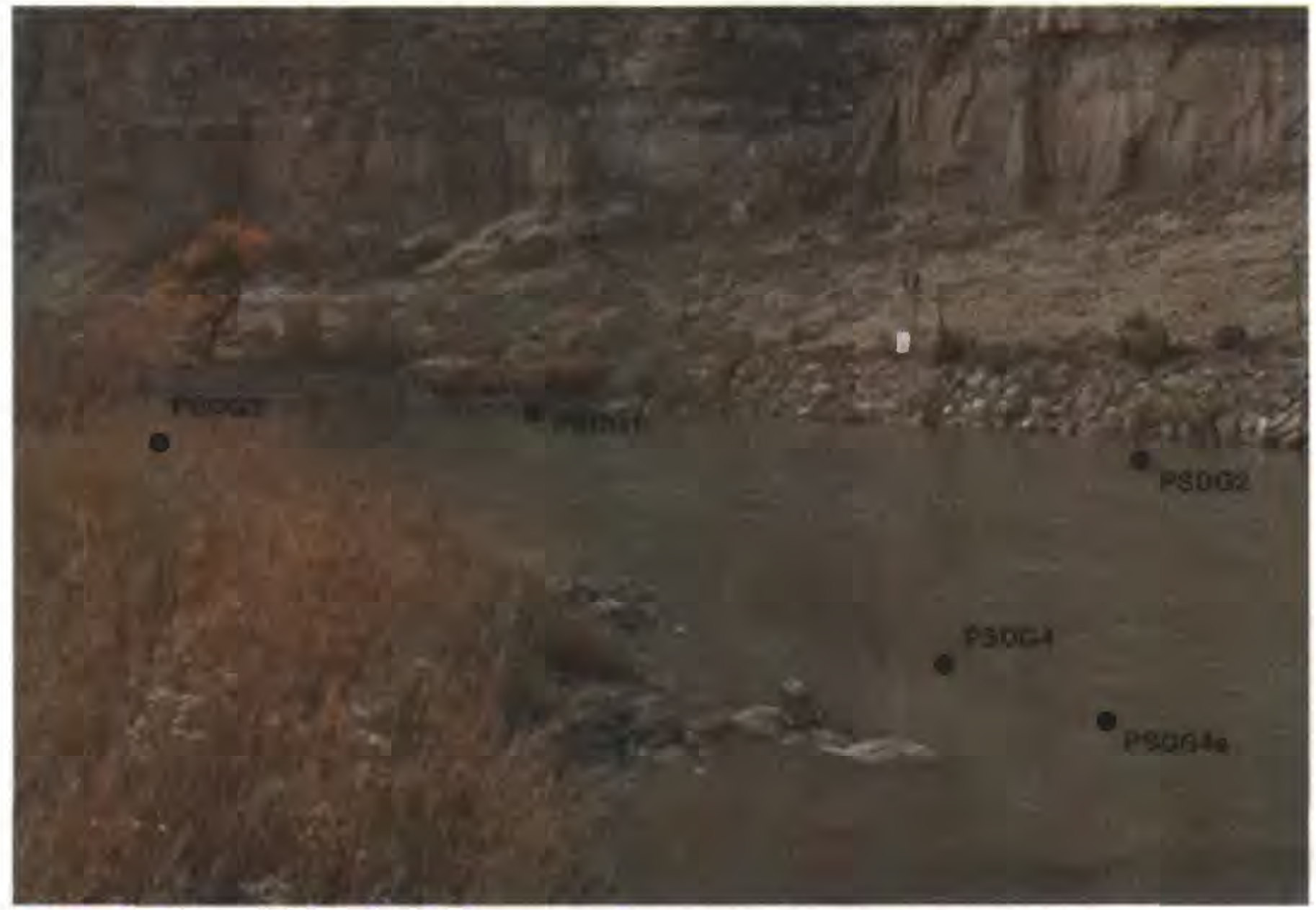

Figure 2. Location of sampling sites upstream from the Price-Stubb Diversion Dam near Palisade, Colorado. Flow is right to left. 
Table 1. List of constituents and results of chemical analyses

[cm, centimeters; ppm, parts per million; Wt., \%, percent by weight; <, less than; ND, not determined]

\begin{tabular}{|c|c|c|c|c|c|c|c|c|}
\hline $\begin{array}{l}\text { Sampling site } \\
\text { (fig. 2) }\end{array}$ & $\begin{array}{l}\text { Sample depth } \\
\text { (cm) }\end{array}$ & $\begin{array}{l}\text { Total silver } \\
\text { (ppm) }\end{array}$ & $\begin{array}{l}\text { Total } \\
\text { copper } \\
\text { (ppm) }\end{array}$ & $\begin{array}{l}\text { Total } \\
\text { lead } \\
\text { (ppm) }\end{array}$ & $\begin{array}{l}\text { Total } \\
\text { zinc } \\
\text { (ppm) }\end{array}$ & $\begin{array}{c}\text { Total } \\
\text { cadmium } \\
\text { (ppm) }\end{array}$ & $\begin{array}{c}\text { Total } \\
\text { chromium } \\
\text { (ppm) }\end{array}$ & $\begin{array}{l}\text { Total } \\
\text { cobalt } \\
\text { (ppm) }\end{array}$ \\
\hline PSDG1 & $0-5 \mathrm{~cm}$ & $<0.5$ & 13 & 20 & 61 & 0.2 & 25 & 6 \\
\hline PSDG1 & $5-10 \mathrm{~cm}$ & $<0.5$ & 13 & 20 & 58 & 0.2 & 26 & 7 \\
\hline PSDG1 & $10-15$ & $<0.5$ & 14 & 21 & 57 & 0.2 & 26 & 7 \\
\hline PSDG1 & $15-20$ & $<0.5$ & 13 & 22 & 52 & 0.2 & 24 & 6 \\
\hline PSDG1 & $20-25$ & $<0.5$ & 14 & 20 & 51 & 0.1 & 25 & 6 \\
\hline PSDG2 & $0-5$ & $<0.5$ & 11 & 23 & 48 & 0.2 & 21 & 6 \\
\hline PSDG2 & $5-8$ & $<0.5$ & 13 & 23 & 61 & 0.2 & 24 & 7 \\
\hline PSDG2 & $8-11$ & $<0.5$ & 15 & 22 & 54 & 0.2 & 24 & 8 \\
\hline PSDG3 & $0-4$ & $<0.5$ & 13 & 42 & 62 & 0.2 & 28 & 6 \\
\hline PSDG3 & $4-6$ & $<0.5$ & 15 & 21 & 61 & 0.2 & 28 & 7 \\
\hline PSDG3 & $6-10$ & $<0.5$ & 14 & 20 & 52 & 0.2 & 26 & 6 \\
\hline PSDG4 & $0-2.5$ & $<0.5$ & 13 & 25 & 63 & 0.2 & 25 & 7 \\
\hline PSDG4 & $2.5-4$ & $<0.5$ & 15 & 22 & 60 & 0.2 & 28 & 7 \\
\hline PSDG4 & $4-6$ & $<0.5$ & 15 & 24 & 59 & 0.2 & 27 & 8 \\
\hline PSDG4a & $0-5$ & $<0.5$ & 13 & 29 & 53 & 0.1 & 25 & 7 \\
\hline PSDG4a & $5-10$ & $<0.5$ & 14 & 23 & 57 & 0.2 & 26 & 7 \\
\hline PSDG4a & $10-15$ & $<0.5$ & 15 & 22 & 57 & 0.2 & 29 & 7 \\
\hline PSDG4a & $15-19$ & $<0.5$ & 14 & 22 & 53 & 0.2 & 27 & 7 \\
\hline \multicolumn{9}{|c|}{ DUPLICATE SAMPLES } \\
\hline DUP PSDG1 & $10-15$ & $<0.5$ & 14 & 23 & 52 & 0.2 & 26 & 7 \\
\hline DUP PSDG3 & $6-10$ & $<0.5$ & 14 & 21 & 53 & 0.2 & 24 & 7 \\
\hline \multicolumn{9}{|c|}{ REFERENCE MATERIALS } \\
\hline \multicolumn{2}{|c|}{$\begin{array}{c}\text { Cody Shale } \\
\text { USGS SCO-1 reported value }\end{array}$} & 0.1 & $29 \pm 2$ & $31 \pm 3$ & $100 \pm 10$ & $0.2 \pm 0.1$ & $68 \pm 5$ & $11 \pm 1$ \\
\hline \multicolumn{2}{|c|}{ USGS laboratory USGS SCO-1 } & $<0.5$ & 26 & 34 & 100 & $<0.1$ & 58 & 13 \\
\hline \multicolumn{2}{|c|}{$\begin{array}{c}\text { Shale } \\
\text { USGS SDO-1 reported value }\end{array}$} & 0.2 & $60 \pm 10$ & $28 \pm 5$ & $64 \pm 7$ & $<2-<10$ & $66 \pm 8$ & $47 \pm 6$ \\
\hline \multicolumn{2}{|c|}{ USGS laboratory USGS SDO-1 } & $<0.5$ & 54 & 30 & 63 & $<0.1$ & 57 & 48 \\
\hline \multicolumn{2}{|c|}{$\begin{array}{c}\text { Marine sediment } \\
\text { USGS MAG-1 reported value }\end{array}$} & $0.08 \pm 0.02$ & $30 \pm 3$ & $24 \pm 3$ & $130 \pm 6$ & $0.2 \pm 0.03$ & $97 \pm 8.0$ & $20 \pm 2$ \\
\hline \multicolumn{2}{|c|}{ USGS laboratory USGS MAG-1 } & $<0.5$ & 26 & 28 & 130 & 0.2 & 94 & 23 \\
\hline \multicolumn{2}{|c|}{$\begin{array}{l}\text { Agricultural soil } \\
\text { NIST } 2709 \text { reported value }\end{array}$} & $<0.5$ & $35 \pm 1$ & $19 \pm 1$ & $\begin{array}{c}106 \pm 3 \\
0\end{array}$ & $0.4 \pm 0.01$ & $130 \pm 4$ & $13 \pm 1$ \\
\hline \multicolumn{2}{|c|}{ USGS laboratory NIST 2709} & $<0.5$ & 32 & 19 & 110 & 0.3 & 100 & 14 \\
\hline
\end{tabular}


Table 1. List of constituents and results of chemical analyses-Continued

\begin{tabular}{|c|c|c|c|c|c|c|c|c|}
\hline $\begin{array}{l}\text { Sampling site } \\
\quad \text { (fig. 2) }\end{array}$ & $\begin{array}{l}\text { Sample depth } \\
\text { (cm) }\end{array}$ & $\begin{array}{l}\text { Total } \\
\text { iron } \\
\text { (Wt. \%) }\end{array}$ & $\begin{array}{c}\text { Total } \\
\text { manga- } \\
\text { nese } \\
(\mathrm{ppm})\end{array}$ & $\begin{array}{c}\text { Total } \\
\text { aluminum } \\
\text { (Wt. \%) }\end{array}$ & $\begin{array}{l}\text { Total } \\
\text { titanium } \\
\text { (Wt. \%) }\end{array}$ & $\begin{array}{c}\text { Total } \\
\text { organic } \\
\text { (Wt. \%) }\end{array}$ & $\begin{array}{l}\text { Total } \\
\text { carbon } \\
\text { (Wt. \%) }\end{array}$ & $\begin{array}{l}\text { Total } \\
\text { sulfur } \\
\text { (Wt. \%) }\end{array}$ \\
\hline PSDG1 & $0-5 \mathrm{~cm}$ & 1.8 & 350 & 4.9 & 0.24 & 0.8 & 1.7 & $<0.1$ \\
\hline PSDG1 & $5-10 \mathrm{~cm}$ & 1.8 & 360 & 4.7 & 0.23 & 0.7 & 1.8 & $<0.1$ \\
\hline PSDG1 & $10-15$ & 1.9 & 380 & 4.9 & 0.25 & 0.5 & 1.5 & $<0.1$ \\
\hline PSDG1 & $15-20$ & 1.7 & 370 & 5.0 & 0.20 & 0.6 & 2.0 & $<0.1$ \\
\hline PSDG1 & $20-25$ & 1.8 & 380 & 5.1 & 0.22 & 1.2 & 2.6 & $<0.1$ \\
\hline PSDG2 & $0-5$ & 1.6 & 320 & 4.4 & 0.23 & 0.3 & 1.2 & $<0.1$ \\
\hline PSDG2 & $5-8$ & 1.8 & 360 & 4.7 & 0.22 & 1.1 & 1.8 & $<0.1$ \\
\hline PSDG2 & $8-11$ & 1.9 & 380 & 5.0 & 0.23 & 1.7 & 3.0 & $<0.1$ \\
\hline PSDG3 & $0-4$ & 1.8 & 320 & 4.7 & 0.24 & 0.7 & 1.7 & $<0.1$ \\
\hline PSDG3 & $4-6$ & 2.0 & 380 & 5.1 & 0.27 & 0.9 & 2.1 & $<0.1$ \\
\hline PSDG3 & $6-10$ & 1.8 & 350 & 4.7 & 0.24 & 1.7 & 2.6 & $<0.1$ \\
\hline PSDG4 & $0-2.5$ & 1.8 & 310 & 4.7 & 0.25 & 0.8 & 1.7 & $<0.1$ \\
\hline PSDG4 & $2.5-4$ & 2.0 & 340 & 5.1 & 0.26 & 0.9 & 2.0 & 0.1 \\
\hline PSDG4 & $4-6$ & 2.0 & 430 & 5.0 & 0.25 & 0.4 & 1.4 & $<0.1$ \\
\hline PSDG4a & $0-5$ & 1.9 & 330 & 4.9 & 0.26 & 0.5 & 1.4 & $<0.1$ \\
\hline PSDG4a & $5-10$ & 2.0 & 350 & 5.0 & 0.25 & 0.7 & 1.8 & $<0.1$ \\
\hline PSDG4a & $10-15$ & 2.1 & 390 & 5.1 & 0.28 & 0.3 & 1.5 & $<0.1$ \\
\hline PSDG4a & $15-19$ & 1.8 & 360 & 4.7 & 0.30 & 0.5 & 1.6 & $<0.1$ \\
\hline \multicolumn{9}{|c|}{ DUPLICATE SAMPLES } \\
\hline DUP PSDG1 & $10-15$ & 1.8 & 370 & 4.9 & 0.25 & 0.5 & 1.4 & $<0.1$ \\
\hline DUP PSDG3 & $6-10$ & 1.8 & 350 & 4.7 & 0.24 & 1.2 & 2.3 & $<0.1$ \\
\hline \multicolumn{9}{|c|}{ REFERENCE MATERIALS } \\
\hline \multicolumn{2}{|c|}{$\begin{array}{l}\text { Cody Shale } \\
\text { USGS SCO-1 reported value }\end{array}$} & $3.6 \pm 0.1$ & $410 \pm 30$ & $7.2 \pm 0.1$ & $0.38 \pm 0.04$ & $0.8 \pm 0.1$ & $0.8 \pm 0.1$ & 0.1 \\
\hline \multicolumn{2}{|c|}{ USGS laboratory USGS SCO-1 } & 3.7 & 420 & 7.3 & 0.34 & 1.1 & 1.1 & \multirow{2}{*}{$\begin{array}{c}0.1 \\
5.4 \pm 0.4\end{array}$} \\
\hline \multicolumn{2}{|c|}{$\begin{array}{c}\text { Shale } \\
\text { USGS SDO- } 1 \text { reported value }\end{array}$} & $6.5 \pm 0.2$ & $320 \pm 40$ & $6.5 \pm 0.1$ & $0.43 \pm 0.02$ & $10 \pm 0.4$ & $10 \pm 0.4$ & \\
\hline \multicolumn{2}{|c|}{ USGS laboratory USGS SDO-1 } & 6.7 & 330 & 6.5 & 0.38 & 9.8 & 9.5 & 5.1 \\
\hline \multicolumn{2}{|c|}{$\begin{array}{c}\text { Marine sediment } \\
\text { USGS MAG-1 reported value }\end{array}$} & $4.8 \pm 0.2$ & $760 \pm 70$ & $8.7 \pm 0.2$ & $0.45 \pm 0.04$ & $2.15 \pm 0.4$ & $2.15 \pm 0.4$ & $0.4 \pm 0.1$ \\
\hline \multicolumn{2}{|c|}{ USGS laboratory USGS MAG-1 } & 5.0 & 730 & 8.6 & 0.42 & 2.3 & 2.2 & 0.2 \\
\hline \multicolumn{2}{|c|}{$\begin{array}{l}\text { Agricultural soil } \\
\text { NIST } 2709 \text { reported value }\end{array}$} & $3.5 \pm 0.1$ & $540 \pm 20$ & $7.5 \pm 0.1$ & $0.34 \pm 0.02$ & 1.2 & 1.2 & 0.1 \\
\hline \multicolumn{2}{|c|}{ USGS laboratory NIST 2709} & 3.5 & 540 & 7.5 & 0.33 & 1.1 & 1.1 & 0.1 \\
\hline
\end{tabular}

FOR MORE INFORMATION, PLEASE CONTACT:

Subdistrict Chief, U.S. Geological Survey

764 Horizon Drive, Room 125, Grand Junction, CO 81506

Telephone: (970) 245-5257 Fax: (970) 245-1026 
Table 1. List of constituents and results of chemical analyses-Continued

\begin{tabular}{|c|c|c|c|c|c|c|c|c|}
\hline $\begin{array}{l}\text { Sampling site } \\
\text { (fig. 2) }\end{array}$ & $\begin{array}{l}\text { Sample depth } \\
\text { (cm) }\end{array}$ & $\begin{array}{l}\text { Total nickel } \\
\text { (ppm) }\end{array}$ & $\begin{array}{c}\text { Total } \\
\text { barium } \\
\text { (ppm) }\end{array}$ & $\begin{array}{c}\text { Total } \\
\text { vanadium } \\
\text { (ppm) }\end{array}$ & $\begin{array}{c}\text { Total } \\
\text { lithium } \\
\text { (ppm) }\end{array}$ & $\begin{array}{c}\text { Total } \\
\text { beryllium } \\
\text { (ppm) }\end{array}$ & $\begin{array}{l}\text { Total molyb- } \\
\text { denum } \\
\text { (ppm) }\end{array}$ & $\begin{array}{c}\text { Total } \\
\text { phos- } \\
\text { phorus } \\
\text { (ppm) }\end{array}$ \\
\hline PSDG1 & $0-5 \mathrm{~cm}$ & 11 & 660 & 57 & 19 & 1.0 & $<5$ & 510 \\
\hline PSDG1 & $5-10 \mathrm{~cm}$ & 12 & 630 & 56 & 19 & 1.0 & $<5$ & 560 \\
\hline PSDG1 & $10-15$ & 13 & 660 & 59 & 18 & 1.1 & $<5$ & 580 \\
\hline PSDG1 & $15-20$ & 12 & 650 & 47 & 26 & 1.0 & $<5$ & 570 \\
\hline PSDG1 & $20-25$ & 13 & 630 & 48 & 31 & 1.1 & $<5$ & 600 \\
\hline PSDG2 & $0-5$ & 9 & 690 & 48 & 15 & 0.9 & $<5$ & 520 \\
\hline PSDG2 & $5-8$ & 13 & 640 & 57 & 18 & 1.0 & $<5$ & 540 \\
\hline PSDG2 & $8-11$ & 13 & 620 & 58 & 18 & 1.0 & $<5$ & 520 \\
\hline PSDG3 & $0-4$ & 11 & 690 & 55 & 17 & 1.0 & $<5$ & 520 \\
\hline PSDG3 & $4-6$ & 14 & 640 & 62 & 21 & 1.1 & $<5$ & 610 \\
\hline PSDG3 & $6-10$ & 12 & 650 & 56 & 18 & 1.0 & $<5$ & 570 \\
\hline PSDG4 & $0-2.5$ & 11 & 610 & 55 & 18 & 1.0 & $<5$ & 500 \\
\hline PSDG4 & $2.5-4$ & 13 & 620 & 61 & 22 & 1.1 & $<5$ & 590 \\
\hline PSDG4 & $4-6$ & 14 & 650 & 61 & 18 & 1.1 & $<5$ & 570 \\
\hline PSDG4a & $0-5$ & 12 & 680 & 58 & 19 & 1.0 & $<5$ & 520 \\
\hline PSDG4a & $5-10$ & 13 & 640 & 60 & 20 & 1.1 & $<5$ & 570 \\
\hline PSDG4a & $10-15$ & 13 & 640 & 63 & 21 & 1.1 & $<5$ & 620 \\
\hline PSDG4a & $15-19$ & 12 & 770 & 57 & 17 & 1.0 & $<5$ & 770 \\
\hline \multicolumn{9}{|c|}{ DUPLICATE SAMPLES } \\
\hline DUP PSDG1 & $10-15$ & 13 & 650 & 59 & 17 & 1.0 & $<5$ & 610 \\
\hline DUP PSDG3 & $6-10$ & 12 & 620 & 56 & 17 & 1.0 & $<5$ & 620 \\
\hline \multicolumn{9}{|c|}{ REFERENCE MATERIALS } \\
\hline \multicolumn{2}{|c|}{$\begin{array}{c}\text { Cody Shale } \\
\text { USGS SCO-1 reported value }\end{array}$} & $24 \pm 4$ & $570 \pm 30$ & $130 \pm 10$ & $45 \pm 3$ & $1.9 \pm 0.1$ & 1 & $900 \pm 90$ \\
\hline \multicolumn{2}{|c|}{ USGS laboratory USGS SCO-1 } & 27 & 560 & 130 & 47 & 1.6 & $<5$ & 870 \\
\hline \multicolumn{2}{|c|}{$\begin{array}{c}\text { Shale } \\
\text { USGS SDO-1 reported value }\end{array}$} & $100 \pm 10$ & $400 \pm 40$ & $160 \pm 20$ & $29 \pm 6$ & $3.3 \pm 0.6$ & $130 \pm 20$ & $480 \pm 31$ \\
\hline \multicolumn{2}{|c|}{ USGS laboratory USGS SDO-1 } & 100 & 49 & 160 & 33 & 2.6 & 160 & 460 \\
\hline \multicolumn{2}{|c|}{$\begin{array}{c}\text { Marine sediment } \\
\text { USGS MAG-1 reported value }\end{array}$} & $53 \pm 8$ & $480 \pm 41$ & $140 \pm 6$ & $79 \pm 4$ & $3.2 \pm 0.4$ & $2 \pm 2$ & $710 \pm 90$ \\
\hline \multicolumn{2}{|c|}{ USGS laboratory USGS MAG-1 } & 52 & 480 & 140 & 79 & 2.5 & $<5$ & 760 \\
\hline \multicolumn{2}{|c|}{$\begin{array}{r}\text { Agricultural soil } \\
\text { NIST } 2709 \text { reported value }\end{array}$} & $88 \pm 5$ & $970 \pm 40$ & $112 \pm 5$ & ND & ND & (2) & $620 \pm 50$ \\
\hline \multicolumn{2}{|c|}{ USGS laboratory NIST 2709} & 85 & 940 & 110 & 57 & 4.0 & $<5$ & 760 \\
\hline
\end{tabular}


Table 1. List of constituents and results of chemical analyses-Continued

\begin{tabular}{|c|c|c|c|c|c|c|c|c|}
\hline $\begin{array}{l}\text { Sampling site } \\
\text { (fig. 2) }\end{array}$ & $\begin{array}{l}\text { Sample depth } \\
(\mathrm{cm})\end{array}$ & $\begin{array}{c}\text { Total } \\
\text { strontium } \\
\text { (ppm) }\end{array}$ & $\begin{array}{c}\text { Total } \\
\text { arsenic } \\
\text { (ppm) }\end{array}$ & $\begin{array}{c}\text { Total } \\
\text { antimony } \\
\text { (ppm) }\end{array}$ & $\begin{array}{c}\text { Total } \\
\text { selenium } \\
\text { (ppm) }\end{array}$ & $\begin{array}{c}\text { Total } \\
\text { mercury } \\
(\mathrm{ppm})\end{array}$ & $\begin{array}{c}\text { Total } \\
\text { thallium } \\
\text { (ppm) }\end{array}$ & $\begin{array}{c}\text { Total } \\
\text { uranium } \\
\text { (ppm) }\end{array}$ \\
\hline PSDG1 & $0-5 \mathrm{~cm}$ & 140 & 4.9 & 0.8 & 0.3 & 0.05 & $<50$ & $<50$ \\
\hline PSDG1 & $5-10 \mathrm{~cm}$ & 140 & 5.2 & 0.8 & 0.3 & 0.04 & $<50$ & $<50$ \\
\hline PSDG1 & $10-15$ & 120 & 5.5 & 0.9 & 0.2 & 0.03 & $<50$ & $<50$ \\
\hline PSDG1 & $15-20$ & 200 & 6.7 & 0.7 & 0.2 & 0.02 & $<50$ & $<50$ \\
\hline PSDG2 & $0-5$ & 120 & 4.6 & 0.7 & 0.2 & 0.01 & $<50$ & $<50$ \\
\hline PSDG2 & $5-8$ & 130 & 5.2 & 0.8 & 0.2 & 0.01 & $<50$ & $<50$ \\
\hline PSDG2 & $8-11$ & 130 & 5.0 & 0.8 & 0.3 & 0.02 & $<50$ & $<50$ \\
\hline PSDG3 & $6-10$ & 130 & 4.9 & 0.9 & 0.3 & 0.02 & $<50$ & $<50$ \\
\hline PSDG4 & $0-2.5$ & 140 & 4.8 & 0.8 & 0.3 & 0.01 & $<50$ & $<50$ \\
\hline PSDG4 & $2.5-4$ & 150 & 5.6 & 0.9 & 0.3 & 0.04 & $<50$ & $<50$ \\
\hline PSDG4 & $4-6$ & 120 & 5.6 & 1.0 & 0.3 & 0.02 & $<50$ & $<50$ \\
\hline PSDG4a & $0-5$ & 140 & 4.8 & 0.8 & 0.2 & 0.02 & $<50$ & $<50$ \\
\hline PSDG4a & $5-10$ & 140 & 5.3 & 0.9 & 0.2 & 0.02 & $<50$ & $<50$ \\
\hline DUP PSDG3 & $6-10$ & 120 & 5.1 & 0.8 & 0.3 & 0.02 & $<50$ & $<50$ \\
\hline \multicolumn{9}{|c|}{ REFERENCE MATERIALS } \\
\hline \multicolumn{2}{|c|}{$\begin{array}{c}\text { Cody Shale } \\
\text { USGS SCO-1 reported value }\end{array}$} & $170 \pm 20$ & $12.4 \pm 1.4$ & $2.5 \pm 0.1$ & $0.9 \pm 0.1$ & 0.05 & 1 & 3 \\
\hline \multicolumn{2}{|c|}{ USGS laboratory USGS SCO-1 } & 160 & 12 & 2.5 & 0.9 & 0.07 & $<50$ & $<50$ \\
\hline \multicolumn{2}{|c|}{ USGS SDO-1 reported value } & $75 \pm 11$ & $69 \pm 9$ & $4.1-4.8$ & $1.9-6.8$ & $0.19 \pm 0.08$ & 8 & $49 \pm 7$ \\
\hline \multicolumn{2}{|c|}{ USGS laboratory USGS SDO-1 } & 71 & 78.0 & 4.1 & 1.6 & 0.16 & $<50$ & $<50$ \\
\hline \multicolumn{2}{|c|}{$\begin{array}{c}\text { Marine sediment } \\
\text { USGS MAG-1 reported value }\end{array}$} & $146 \pm 15$ & $9.2 \pm 1.2$ & $1.0 \pm 0.1$ & $1.2 \pm 0.1$ & $(0.02)$ & $<50$ & $<50$ \\
\hline \multicolumn{2}{|c|}{ USGS laboratory USGS MAG-1 } & 130 & 9.0 & 0.9 & 1.2 & 0.05 & $<50$ & $<50$ \\
\hline \multicolumn{2}{|c|}{$\begin{array}{r}\text { Agricultural soil } \\
\text { NIST } 2709 \text { reported value }\end{array}$} & $230 \pm 2.0$ & $18 \pm 1$ & $7.9 \pm 0.6$ & $1.6 \pm 0.1$ & $1.40 \pm 0.08$ & (1) & (3) \\
\hline \multicolumn{2}{|c|}{ USGS laboratory NIST 2709} & 210 & 18 & 6.2 & 1.5 & 1.4 & $<50$ & $<50$ \\
\hline
\end{tabular}

